\title{
Statistical Estimation
}

National Cancer Institute

\section{Source}

National Cancer Institute. Statistical Estimation. NCI Thesaurus. Code C53327.

A statistical procedure for assig ning a plausable value to a parameter based on measured/empirical data. 